Bull. Korean Math. Soc. 51 (2014), No. 5, pp. 1259-1267

http://dx.doi.org/10.4134/BKMS.2014.51.5.1259

\title{
HYPERBOLICITY OF CHAIN TRANSITIVE SETS WITH LIMIT SHADOWING
}

\author{
Abbas Fakhari, Seunghee Lee, and Khosro Tajbakhsh
}

\begin{abstract}
In this paper we show that any chain transitive set of a diffeomorphism on a compact $C^{\infty}$-manifold which is $C^{1}$-stably limit shadowable is hyperbolic. Moreover, it is proved that a locally maximal chain transitive set of a $C^{1}$-generic diffeomorphism is hyperbolic if and only if it is limit shadowable.
\end{abstract}

Transitive sets, homoclinic classes and chain components of a diffeomorphism are natural candidates to replace the hyperbolic basic sets in nonhyperbolic theory of differentiable dynamical systems, and many recent papers explored their "hyperbolic-like" properties (for more details, see $[2,6,8,9,14,15]$ ).

In this paper we study the chain transitive sets which are limit shadowable. Let us be more precise. Let $M$ be a compact $C^{\infty}$-manifold, and let Diff(M) be the space of diffeomorphisms of $M$ endowed with the $C^{1}$-topology. Denote by $d$ the distance on $M$ induced from a Riemannian metric on the tangent bundle $T M$. For $\delta>0$, a sequence $\left\{x_{n}\right\}_{n \in \mathbb{Z}}$ in $\Lambda$ is called $a \delta$-limit chain if

$$
\lim _{|n| \rightarrow \infty} d\left(f\left(x_{n}\right), x_{n+1}\right)=0 \text { and } d\left(f\left(x_{n}\right), x_{n+1}\right)<\delta
$$

for any $n \in \mathbb{Z}$. For a closed $f$-invariant set $\Lambda \subset M$, we say that $f$ has the limit shadowing property in $\Lambda$ (or $\Lambda$ is limit shadowable for $f$ ) if there exists $\delta>0$ such that for any $\delta$-limit chain $\xi=\left\{x_{n}\right\}_{n \in \mathbb{Z}}$ in $\Lambda$ there exists $y \in \Lambda$ satisfying

$$
d\left(f^{n}(y), x_{n}\right) \rightarrow 0 \text { as }|n| \rightarrow \infty .
$$

We also say that the $\delta$-limit chain $\xi$ is limit shadowed by the point $y$. Note that the limit shadowing property does not imply the shadowing property. For example, let $f$ be a diffeomorphism on the unit circle $\mathbb{S}^{1}$ with coordinates $x \in[0,1)$ which has only three fixed points $0, \frac{1}{3}$ and $\frac{2}{3}$ such that 0 is source, $\frac{2}{3}$ is sink and $\frac{1}{3}$ is nonhyperbolic. Then it is clear that $f$ has the limit shadowing property in $\mathbb{S}^{1}$ but it does not satisfy the shadowing property.

Received June 2, 2011; Revised April 20, 2013.

2010 Mathematics Subject Classification. Primary 58B34, 58J42, 81T75.

Key words and phrases. noncommutative complex torus, mirror symmetry, Kronecker foliation.

This work was financially supported by KRF 2003-041-C20009. 
Definition. Let $\Lambda$ be an invariant set for $f \in \operatorname{Diff}(\mathrm{M})$. We say that $\Lambda$ admits a dominated splitting if the tangent bundle $T_{\Lambda} M$ has a $D f$-invariant splitting $E \oplus F$ such that for some $C>0$ and $0<\lambda<1$,

$$
\left\|\left.D f^{n}\right|_{E(x)}\right\| \cdot\left\|\left.D f^{-n}\right|_{F\left(f^{n}(x)\right)}\right\| \leq C \lambda^{n}
$$

for all $x \in \Lambda$ and $n \geq 0$. The set $\Lambda$ is hyperbolic if the subbundle $E$ is uniformly contracting and the subbundle $F$ is uniformly expanding; i.e., for some $C>0$ and $0<\lambda<1$,

$$
\left\|\left.D f^{n}\right|_{E^{s}(x)}\right\| \leq C \lambda^{n} \text { and }\left\|\left.D f^{-n}\right|_{E^{u}\left(f^{n}(x)\right)}\right\| \leq C \lambda^{n}
$$

for all $x \in \Lambda$ and $n \geq 0$.

Let $P_{h}(f)$ be the set of all hyperbolic periodic points of $f$. It is well known that for any $p \in P_{h}(f)$ with period $k$, the sets

$$
\begin{gathered}
W^{s}(p)=\left\{x \in M: f^{k n}(x) \rightarrow p \text { as } n \rightarrow \infty\right\}, \text { and } \\
W^{u}(p)=\left\{x \in M: f^{-k n}(x) \rightarrow p \text { as } n \rightarrow \infty\right\}
\end{gathered}
$$

are $C^{1}$-injectively immersed submanifolds of $M$. A point $x \in W^{s}(p) \cap W^{u}(p)$ is called a homoclinic point of $f$ associated to $p$, and it is said to be a transversal homoclinic point of $f$ if the above intersection is transversal at $x$; i.e., $x \in$ $W^{s}(p) \pitchfork W^{u}(p)$. The closure of the transversal homoclinic points of $f$ associated to the orbit of $p$ is called the homoclinic class of $f$ associated to $p$ and denoted by $H_{f}(p)$. Let $q$ be another hyperbolic periodic point of $f$. The two points $p$ and $q$ are called homoclinically related, and write $p \sim q$ if

$$
W^{s}(p) \bar{\pitchfork} W^{u}(q) \neq \emptyset \text { and } W^{u}(p) \pitchfork W^{s}(q) \neq \emptyset \text {. }
$$

By Smale's transverse homoclinic point theorem, $H_{f}(p)$ coincides with the closure of the set of hyperbolic periodic points $q$ of $f$ such that $p \sim q$. Note that if $p$ is a hyperbolic periodic point of $f$, then there is a neighborhood $U$ of $p$ and a $C^{1}$-neighborhood $\mathcal{U}(f)$ of $f$ such that for any $g \in \mathcal{U}(f)$ there exists a unique hyperbolic periodic point $p_{g}$ of $g$ in $U$ with the same period and the same index as those of $p$. The point $p_{g}$ is called the continuation of $p$.

Definition. We say that $f$ has the $C^{1}$-stably limit shadowing property in $\Lambda$ (or $\Lambda$ is $C^{1}$-stably limit shadowable for $f$ ) if there are a $C^{1}$-neighborhood $\mathcal{U}(f)$ of $f$ and a compact neighborhood $U$ of $\Lambda$ such that

(1) $\Lambda=\Lambda_{f}(U)=\bigcap_{n \in \mathbb{Z}} f^{n}(U)$; i.e., $\Lambda$ is locally maximal in $U$,

(2) $\Lambda_{g}$ is limit shadowable for $g \in \mathcal{U}(f)$,

where $\Lambda_{g}=\bigcap_{n \in \mathbb{Z}} g^{n}(U)$ is the continuation of $\Lambda=\Lambda_{f}(U)$. In this case, we say that $\Lambda$ is $C^{1}$-stably limit shadowable with respect to $U$ and $\mathcal{U}(f)$.

It is known that any locally maximal hyperbolic set is $C^{1}$-stably limit shadowable (see [13]).

For given $\delta>0$, a sequence $\left\{x_{i}\right\}_{i=a}^{b}(-\infty \leq a<b \leq \infty)$, is called $a \delta$-pseudo orbit of $f$ if $d\left(f\left(x_{i}\right), x_{i+1}\right)<\delta$ for all $a \leq i \leq b-1$. A closed $f$-invariant set 
$\Lambda \subset M$ is chain transitive if for any two points $x, y \in \Lambda$ and any $\delta>0$, there is a finite $\delta$-pseudo orbit $\left\{x_{i}\right\}_{i=a}^{b} \subset \Lambda$ of $f$ with $x_{a}=x$ and $x_{b}=y$.

In this paper, we first study the hyperbolicity of a chain transitive set by making use of the limit shadowing property under $C^{1}$-open condition. More precisely, we have the following theorem.

Theorem A. A chain transitive set $\Lambda$ is $C^{1}$-stably limit shadowable if and only if it is hyperbolic.

A subset $\mathcal{R} \subset \operatorname{Diff}^{\mathrm{r}}(\mathrm{M})(\mathrm{r} \geq 1)$ is called residual if it contains the intersection of a countable family of open and dense subsets of $\operatorname{Diff}^{\mathrm{r}}(\mathrm{M})$. A property " $\mathcal{P}$ " is said to be $C^{r}$-generic if " $\mathcal{P}$ " holds for all diffeomorphisms in a residual subset of $\operatorname{Diff}^{\mathrm{r}}(\mathrm{M})$.

Recently, Abdenur and Díaz in [2] obtained a necessary and sufficient condition for a locally maximal transitive set $\Lambda$ of a $C^{1}$-generic diffeomorphism $f$ to be hyperbolic as follow: either $\Lambda$ is hyperbolic, or there are a $C^{1}$-neighborhood $\mathcal{U}(f)$ of $f$ and a neighborhood $V$ of $\Lambda$ such that every $g \in \mathcal{U}(f)$ does not have the shadowing property on $V$.

As a second result of the paper, we get the following result for the limit shadowable chain transitive sets.

Theorem B. There exists a residual subset $\mathcal{R}$ of $\operatorname{Diff}^{1}(M)$ such that a locally maximal chain transitive set $\Lambda$ of $f \in \mathcal{R}$ is limit shadowable if and only if it is hyperbolic.

\section{Proof of Theorem A}

The next lemma, known as Franks' Lemma, is a simple yet powerful result allowing us to perturb the tangent map along a finite set with an arbitrarily small support.

Lemma 1.1 (Franks' Lemma). Let $\mathcal{U}(f)$ be any given $C^{1}$-neighbor-hood of $f$. Then there exist $\epsilon>0$ and a $C^{1}$-neighborhood $\mathcal{U}_{0}(f)$ of $\mathcal{U}(f)$ of $f$ such that for given $g \in \mathcal{U}_{0}(f)$, a finite set $\left\{x_{1}, x_{2}, \ldots, x_{N}\right\}$, a neighborhood $U$ of $\left\{x_{1}, x_{2}, \ldots, x_{N}\right\}$ and linear maps $L_{i}: T_{x_{i}} M \rightarrow T_{g\left(x_{i}\right)} M$ satisfying $\| L_{i}-$ $D_{x_{i}} g \| \leq \epsilon$ for all $1 \leq i \leq N$, there exists $\tilde{g} \in \mathcal{U}(f)$ such that $\tilde{g}(x)=g(x)$ if $x \in\left\{x_{1}, x_{2}, \ldots, x_{N}\right\} \cup(M \backslash U)$ and $D_{x_{i}} \tilde{g}=L_{i}$ for all $1 \leq i \leq N$.

In this section, we will prove Theorem A by using the technique developed by Mañé in [11]. To use it, we need the following lemma.

Lemma 1.2. If $f$ is $C^{1}$-stably limit shadowable in a chain transitive set $\Lambda$ with respect to a neighborhood $U$ of $\Lambda$ and a $C^{1}$-neighborhood $\mathcal{U}(f)$ of $f$, then any periodic point of $g \in \mathcal{U}(f)$ in $\Lambda_{g}$ is hyperbolic.

Proof. Let $\epsilon>0$ and $\mathcal{U}_{0}(f) \subset \mathcal{U}(f)$ are given by Lemma 1.1. Suppose that there exists a non-hyperbolic periodic point $q \in \Lambda_{g}$ for some $g \in \mathcal{U}_{0}(f)$ (since $\Lambda$ is locally maximal, reducing $\mathcal{U}(f)$ if necessary, we may assume that $q$ is 
contained in the interior of $U)$. To simplify the notations, we assume that $g(q)=q$ (other case is similar). Then the use of Lemma 1.1, one may construct a diffeomorphism $g_{1} \in \mathcal{U}_{0}(f) C^{1}$-nearby $g$ possessing either

(i) a $g_{1}$-invariant normally hyperbolic small $\operatorname{arc} \mathcal{I}_{q} \subset U$ such that $\left.g_{1}\right|_{\mathcal{I}_{q}} ^{k}=$ $i d$ for some $k>0$; or

(ii) a $g_{1}$-invariant normally hyperbolic small circle $\mathcal{C}_{q} \subset U$ with a small diameter and center at $q$ such that $\left.g_{1}\right|_{\mathcal{C}_{q}}$ is conjugated to an irrational rotation.

Since $\mathcal{I}_{q}$ and $\mathcal{C}_{1}$ are $g_{1}$-invariant, we see that $\mathcal{I}_{q} \subset \Lambda_{g}$ and $\mathcal{C}_{q} \subset \Lambda_{g}$. Since $g_{1}$ has the limit shadowing property on $\Lambda_{g}$, both $\left.g_{1}^{k}\right|_{\mathcal{I}_{q}}$ and $\left.g_{1}\right|_{\mathcal{C}_{q}}$ must have the limit shadowing property. By the $C^{1}$-stablity of limit shadowing, this is a contradiction. This completes the proof of Lemma 1.2.

Lemma 1.3. If $f$ has the limit shadowing property in a chain transitive set $\Lambda$, then for any $p, q \in \Lambda \cap P_{h}(f)$,

$$
W^{s}(p) \cap W^{u}(q) \neq \emptyset \text { and } W^{u}(p) \cap W^{s}(q) \neq \emptyset .
$$

Proof. Let $p$ and $q$ be two periodic points of $f$ in $\Lambda$. Take a constant $\delta>0$ such that every $\delta$-pseudo orbit in $\Lambda$ is limit shadowed by a point in $\Lambda$. Since $\Lambda$ is chain transitive, there is a $\delta$-pseudo orbit $\left\{x_{0}=p, x_{1}, \ldots, x_{n}=q\right\}$ in $\Lambda$. Construct a sequence

$$
\xi=\left\{\ldots, p, p, x_{0}, x_{1}, \ldots, x_{n}, q, q, \ldots\right\} .
$$

Then $\xi$ is a $\delta$-limit chain in $\Lambda$. Since $\Lambda$ is limit shadowable for $f$, we can choose a point $y \in \Lambda$ such that

$$
d\left(f^{n}(y), x_{n}\right) \rightarrow 0 \text { as }|n| \rightarrow \infty .
$$

For $\eta>0$, we can choose $n_{1}>0$ sufficiently large such that

$$
f^{-n}(y) \in W_{\eta}^{u}(p) \quad \text { and } \quad f^{n}(y) \in W_{\eta}^{s}(q)
$$

for all $n \geq n_{1}$. Therefore

$$
y \in f^{n}\left(W_{\eta}^{u}(p)\right) \quad \text { and } \quad y \in f^{-n}\left(W_{\eta}^{s}(q)\right) .
$$

Thus $y \in W^{u}(p) \cap W^{s}(q)$, and so $W^{u}(p) \cap W^{s}(q) \neq \emptyset$. Similarly we can show that $W^{u}(q) \cap W^{s}(p) \neq \emptyset$.

Remark 1.4. Using the two lemmas above one can deduce that the index of periodic points in $\Lambda$ doesn't change. In fact, if there are two periodic points $p$ and $q$ in $\Lambda$ with different indices, then the same happens for a Kupka-Smale diffeomorphism $g$ sufficiently close to $f$. On the other hand, by Lemma 1.3, stable and unstable manifolds of $p_{g}$ and $q_{q}$, the continuation of $p$ and $q$ respectively, should intersect each other. This contradicts to $g$ being Kupka-Smale.

By Proposition II.1 in [11] and two lemma above we have the following proposition which plays an essential rule in our proof. 
Proposition 1.5. If a chain transitive set $\Lambda$ is $C^{1}$-stably limit shadowable, then there exist a neighborhood $\mathcal{U}_{0}(f)$ of $f$, a constant $0<\lambda<1$ and a natural number $m>0$ such that

(1) for any $g \in \mathcal{U}_{0}(f)$, if $q \in \Lambda_{g}$ is a periodic point of $g$ with period $\pi(q)$, then

$$
\begin{aligned}
& \prod_{i=0}^{\pi(q)-1}\left\|\left.D_{g^{i m}(q)} g^{m}\right|_{E^{s}\left(g^{i m}(q)\right)}\right\|<\lambda^{\pi(q)} \text { and } \\
& \prod_{i=0}^{\pi(q)-1}\left\|\left.D_{g^{-i m}(q)} g^{-m}\right|_{E^{s}\left(g^{i m}(q)\right)}\right\|<\lambda^{\pi(q)}
\end{aligned}
$$

(2) $\overline{\operatorname{Per}(g)}$ admits a dominated splitting $E \oplus F$ with $\operatorname{dim} E=\operatorname{index}(p)$.

Before proving the hyperbolicity of $\Lambda$, we recall the Mañé's Ergodic Closing Lemma obtained in [11]. Denote by $B_{\epsilon}(f, x)$ an $\epsilon$-tubular neighborhood of the $f$-orbit of $x$; that is,

$$
B_{\epsilon}(f, x)=\left\{y \in M: d\left(f^{n}(x), y\right)<\epsilon \text { for some } n \in \mathbb{Z}\right\} .
$$

We say that a point $x \in M$ is well closable for $f \in \operatorname{Diff}(\mathrm{M})$ if for any $\epsilon>0$ there are $g \in \operatorname{Diff}(\mathrm{M})$ with $d_{1}(f, g)<\epsilon$ and $p \in M$ such that $p \in P(g), g=f$ on $M-B_{\epsilon}(x, f)$ and $d\left(f^{n}(x), g^{n}(p)\right) \leq \epsilon$ for any $0 \leq n \leq \pi(p)$ and $d_{1}$ is the $C^{1}$-metric. Let $\Sigma_{f}$ denote the set of well closable points of $f$. Then we can state the Closing Lemma as follows.

Lemma 1.6 (Mañé Ergodic Closing Lemma, [11]). For any $f$-invariant probability measure $\mu$ on $M$, we have $\mu\left(\Sigma_{f}\right)=1$.

To complete the proof of Theorem A, it is sufficient to show the following.

Theorem 1.7. The set $\overline{\operatorname{Per}(f)}$ is hyperbolic.

Proof. By Proposition 1.5(2), we know that $\overline{\operatorname{Per}(f)}$ admits a dominated splitting $E \oplus F$ with $\operatorname{dim} E=i n d e x(p)$. We will show the hyperbolicity of direction $E$, the other one can be treated similarly. To show this, it is enough to prove that

$$
\liminf _{n \rightarrow \infty}\left\|\left.D f^{n}\right|_{E(x)}\right\|=0
$$

for all $x \in \overline{\operatorname{Per}(f)}$. Suppose not. Then by Birkhooff's Theorem and Mañé's Ergodic Closing Lemma, we can find a point $z \in \Lambda \cap \Sigma_{f}$ such that

$$
\lim _{n \rightarrow \infty} \frac{1}{n} \sum_{i=0}^{n-1} \log \left\|\left.D_{f^{i m}(z)} f^{m}\right|_{E\left(f^{i m}(z)\right)}\right\| \geq 0 .
$$

By Proposition 1.5, $z$ can not be a periodic point of $f$. Let $m$ and $0<\lambda<1$ be given by Proposition 1.5, and take $\lambda<\lambda_{0}<1$ and $n_{0}>0$ such that

$$
\frac{1}{n} \sum_{i=0}^{n-1} \log \left\|\left.D_{f^{i m}(z)} f^{m}\right|_{E\left(f^{i m}(z)\right)}\right\| \geq \log \lambda_{0}
$$


for $n>n_{0}$. By Mañé's Ergodic Closing Lemma, we may find $\tilde{g}$ in a connected neighborhood $\mathcal{U}_{0}(f)$ of $f$ and $\tilde{z} \in \Lambda_{\tilde{g}} \cap P(\tilde{g})$ nearby $z$ such that

$$
\lambda_{0}^{\pi(\tilde{z})} \leq \prod_{i=0}^{\pi(\tilde{z})-1}\left\|\left.D_{\tilde{g}^{i m}(\tilde{z})} \tilde{g}^{m}\right|_{E\left(\tilde{g}^{i m}(\tilde{z})\right)}\right\| .
$$

On the other hand, by Proposition 1.5, we see that

$$
\prod_{i=0}^{\pi(\tilde{z})-1}\left\|\left.D_{\tilde{g}^{i m}(\tilde{z})} \tilde{g}^{m}\right|_{E\left(\tilde{g}^{i m}(\tilde{z})\right)}\right\|<\lambda^{\pi(\tilde{z})} .
$$

This contradicts (1), and so $E$ is uniformly contracting under $D f$.

\section{Proof of Theorem B}

We begin the section by a remark assembling the behavior of a Kupka-Smale diffeomorphism with the limit shadowing.

Remark 2.1. As mentioned in the previous section, if $f$ has limit shadowing on $\Lambda$, then any two periodic points $p$ and $q$ in $\Lambda$ have the same index and their stable and unstable manifolds cut each other. If $f$ is a Kupka-Smale diffeomorphism, then one can ensure the intersections are transverse. In other word, in this case $p \sim q$.

Lemma 2.2 ([10, Lemma 2.2]). There is a residual set $\mathcal{R}_{0} \subset \operatorname{Diff}(M)$ such that every $f \in \mathcal{R}_{0}$ satisfies the following property: For any closed $f$-invariant set $\Lambda \subset M$, if there are a sequence of diffeomorphisms $f_{n}$ converging to $f$ and a sequence of hyperbolic periodic orbits $P_{n}$ of $f_{n}$ with index $k$ verifying $\lim _{n \rightarrow \infty} P_{n}=\Lambda$, then there is a sequence of hyperbolic periodic orbits $Q_{n}$ of $f$ with index $k$ such that $\Lambda$ is the Hausdorff limit of $Q_{n}$, where the index of a hyperbolic periodic orbit $P$ is the dimension of the stable manifold of $P$.

To complete the proof of Theorem B, we let $\mathcal{R}=\mathcal{R}_{0} \cap \mathcal{K S}$, where $\mathcal{K S}$ denotes the set of Kupka-Smale diffeomorphisms. The following proposition is crucial in the proof of the theorem.

Proposition 2.3. Let $f \in \mathcal{R}$, and let $\Lambda$ be a limit shadowable chain transitive set of $f$ which is locally maximal. Then there exist constants $m>0$ and $0<\lambda<1$ such that for any periodic point $p \in \Lambda$,

$$
\begin{aligned}
& \prod_{i=0}^{\pi(p)-1}\left\|\left.D f^{m}\right|_{E^{s}\left(f^{i m}(p)\right)}\right\|<\lambda^{\pi(p)}, \\
& \prod_{i=0}^{\pi(p)-1}\left\|\left.D f^{-m}\right|_{E^{u} f^{-m}(p)}\right\|<\lambda^{\pi(p)}
\end{aligned}
$$

and

$$
\left\|\left.D f^{m}\right|_{E^{s}(p)}\right\| \cdot\left\|\left.D f^{-m}\right|_{E^{u} f^{m}(p)}\right\|<\lambda^{2} .
$$


Proof. Since $f \in \mathcal{R}_{0}$, all periodic points in $\Lambda$ have the same index and $\Lambda$ is locally maximal, we can choose a $C^{1}$-neighborhood $\mathcal{U}(f)$ of $f$ and a neighborhood $U$ of $\Lambda$ such that every $g \in \mathcal{U}(f)$ has no nonhyperbolic periodic orbit which is contained in $U$. In fact, if there are non-hyperbolic periodic points, then by using Franks Lemma one can produce periodic points of different index in $U$ for diffeomorphisms sufficiently close to $f$. Since $f \in \mathcal{R}$, the same holds for $f$. We arrive at the contradiction by Remark 2.1.

By applying Lemma II.3 in [11], we get constants $K>0, m_{0} \in \mathbb{Z}^{+}$and $0<\lambda<1$ such that for any periodic point $p \in \Lambda$ with $\pi(p) \geq K$,

$$
\begin{aligned}
& \prod_{i=0}^{\pi(p)-1}\left\|\left.D f^{m_{0}}\right|_{E^{s}\left(f^{\left.i m_{0}(p)\right)}\right.}\right\|<\lambda^{\pi(p)}, \\
& \prod_{i=0}^{\pi(p)-1}\left\|\left.D f^{-m_{0}}\right|_{E^{u} f^{-m_{0}(p)}}\right\|<\lambda^{\pi(p)}
\end{aligned}
$$

and

$$
\left\|\left.D f^{m_{0}}\right|_{E^{s}(p)}\right\| \cdot\left\|\left.D f^{-m_{0}}\right|_{E^{u}\left(f^{m}(p)\right)}\right\|<\lambda^{2} .
$$

Let $\Lambda_{0}$ be the set of all periodic points in $\Lambda$ whose periods are less than $K$. Since every periodic point of $f$ is hyperbolic, there are only a finite number of periodic points in $\Lambda_{0}$, and so $\Lambda_{0}$ is hyperbolic for $f$. Let $k$ be a positive integer such that

$$
\left\|\left.D f^{k m_{0}}\right|_{E^{s}(x)}\right\|<\lambda \text { and }\left\|\left.D f^{-k m_{0}}\right|_{E^{u}(x)}\right\|<\lambda
$$

for all $x \in \Lambda_{0}$. If we let $m=k m_{0}$, then we know that $m$ and $\lambda$ are the required constants satisfying Proposition 2.3.

For any periodic point $p$ of a diffeomorphism $f$, we can see that $\mu_{p}$ given by

$$
\mu_{p}=\frac{1}{\pi(p)} \sum_{i=0}^{\pi(p)-1} \delta_{f^{i}(p)}
$$

is a $f$-invariant ergodic probability measure concentrated on $M$. Finally we will use the following proposition comes from the Mane's ergodic closing lemma in [11] which gives the measure theoretical viewpoint of the approximation by periodic orbits.

Proposition 2.4. There exists a residual subset $\mathcal{R}_{1}$ of $\operatorname{Diff}^{1}(\mathrm{M})$ such that for any $f \in \mathcal{R}_{1}$ and for any $f$-invariant ergodic probability measure $\mu$ of $f$ there is a sequence of hyperobolic periodic points $p_{n}$ such that

- $\mu_{p_{n}} \rightarrow \mu$ in weak* topology,

- $\mathcal{O}(p) \rightarrow \operatorname{Supp}(\mu)$ in Hausdorff metric.

Proof of Theorem B. Put $\mathcal{R}_{2}=\mathcal{R} \cap \mathcal{R}_{1}$, and let $f \in \mathcal{R}_{2}$ has the limit shadowing property in $\Lambda$. Now we will prove that the dominated splitting $E \oplus F$ given by Proposition 2.3 is in fact hyperbolic. First we show that $E$ is uniformly 
contracting under $D f$. To this end, it suffices by using Lemma I.5 in [12], to prove that

$$
\int \log \left(\left\|\left.D f^{m}\right|_{E(x)}\right\|\right) d \mu<0
$$

for every $f$-invariant ergodic probability measure $\mu$. Since $f \in \mathcal{R}_{1}$ there exists a sequence $\mathcal{O}\left(p_{n}\right)$ of periodic orbits of $f$ with $\mathcal{O}\left(p_{n}\right) \rightarrow \Lambda$ in the Hausdorff topology and periodic measures $\mu_{p_{n}}$ concentrated on the orbit of $p_{n}$ converges to $\mu_{0}$ in weak*-topology. By the local maximality of $\Lambda$, for sufficiently large $n$, the periodic orbits $\mathcal{O}\left(p_{n}\right)$ are contained in $\Lambda$. On the other hand, if we apply Proposition 2.3, then we have

$$
\int \log \left(\left\|\left.D f^{m}\right|_{E(x)}\right\|\right) d \mu_{n}<\log \lambda
$$

for sufficiently large $n$. Since $\mu_{n}$ converges to $\mu_{0}$ in the weak ${ }^{*}$ topology, we have

$$
\int \log \left(\left\|\left.D f^{m}\right|_{E(x)}\right\|\right) d \mu_{n} \rightarrow \int \log \left(\left\|\left.D f^{m}\right|_{E(x)}\right\|\right) d \mu_{0}
$$

as $n \rightarrow \infty$. Hence we get $\int \log \left(\left\|\left.D f^{m}\right|_{E(x)}\right\|\right) d \mu_{0}<0$. The contradiction proves that $D f$ is contracting on $E$. Similarly, one can show that $D f$ is expanding on $F$.

\section{References}

[1] F. Abdenur, C. Bonatti, and S. Crovisier, Nonuniform hyperbolicity for $C^{1}$-generic diffeomorphisms, Israel J. Math. 183 (2011), 1-60.

[2] F. Abdenur and L. J. Díaz, Pseudo-orbit shadowing in the $C^{1}$ topology, Discrete Contin. Dyn. Syst. 17 (2007), no. 2, 223-245.

[3] C. Bonatti, L. J. Diaz, and M. Vianan, Dynamics beyond uniform hyperbolicity, A global geometric and probabilistic perspective, Encyclopedia of Mathematical Sciences, 102 Mathematical Physics, III. Springer-verlag, Berlin, 2005.

[4] C. Bonattio, S. Gan, and D. Yang, On the hyperoblicity of homoclinic classes, Discrete Contin. Dyn. Syst. 25 (2009), no. 4, 1143-1162.

[5] S. Crovisier, Periodic orbits and chain transitive sets of $C^{1}$-diffeomorphisms, Publ. Math. Inst. Hautes Études Sci. 104 (2006), 87-141.

[6] A. Fakhari, K. Lee, and A. Tajbakhsh, Diffeomorphisms with $\mathcal{L}_{p}$-shadowing property, Acta Math. Sin. (Engl. Ser.) 27 (2011), no. 1, 19-28.

[7] J. Franks, Necessary conditions for stability of diffeomorphisms, Trans. Amer. Math. Soc. 158 (1971), 301-308.

[8] K. Lee, Hyperbolic sets with the strong limit shadowing property, J. Inequal. Appl. 6 (2001), no. 5, 507-517.

[9] K. Lee, K. Moriyasu, and K. Sakai, $C^{1}$-stable shadowing diffeomorphism, Discrete Contin. Dyn. Syst. 22 (2008), no. 3, 683-697.

[10] K. Lee and X. Wen, Shadowable chain transitive sets of $C^{1}$-generic diffeomorphisms, Bull. Korean Math. Soc. 49 (2012), no. 2, 263-270.

[11] R. Mañé, An ergodic closing lemma, Ann. of Math. 16 (1982), no. 3, 503-540.

[12] $ـ$ A proof of the $C^{1}$ stability conjecture, Inst. Hautes Études Sci. Publ. Math. 66 (1988), 161-210.

[13] S. Yu. Pilyugin, Shadowing in Dynamical Systems, Lecture Notes in Math. 1706, Spinger-Verlag, Berlin, 1999. 
HYPERBOLICITY OF CHAIN TRANSITIVE SETS WITH LIMIT SHADOWING 1267

[14] - Sets of dynamical systems with various limit shadowing properties, J. Dynam. Differential Equations 19 (2007), no. 3, 747-775.

[15] K. Sakai, $C^{1}$-stably shadowable chain components, Ergodic Theory Dyanam. Systems 28 (2008), no. 3, 987-1029.

ABBAS FAKHARI

Department of Mathematics

Shahid Beheshti University

G. C. Tehran 19839, Iran

E-mail address: a_fakhari@sbu.ac.ir

Seunghee Lee

Department of Mathematics

Chungnam National University

DAEJEON 305-764, KoREA

E-mail address: shlee@cnu.ac.kr

KhosRo TAJBAKHSH

Department of Mathematics

Faculty of Mathematical Sciences

Tarbiat Modares University

14115-134, TeHran, Iran

E-mail address: khtajbakhsh@modares.ac.ir 